\title{
Rarest First and Choke Algorithms Are Enough
}

\author{
Arnaud Legout \\ I.N.R.I.A \\ Sophia Antipolis France \\ arnaud.legout@sophia.inria.fr
}

\author{
G. Urvoy-Keller and P. Michiardi \\ Institut Eurecom \\ Sophia Antipolis France \\ \{Guillaume.Urvoy,Pietro.Michiardi\}@eurecom.fr
}

\begin{abstract}
The performance of peer-to-peer file replication comes from its piece and peer selection strategies. Two such strategies have been introduced by the BitTorrent protocol: the rarest first and choke algorithms. Whereas it is commonly admitted that BitTorrent performs well, recent studies have proposed the replacement of the rarest first and choke algorithms in order to improve efficiency and fairness. In this paper, we use results from real experiments to advocate that the replacement of the rarest first and choke algorithms cannot be justified in the context of peer-to-peer file replication in the Internet.

We instrumented a BitTorrent client and ran experiments on real torrents with different characteristics. Our experimental evaluation is peer oriented, instead of tracker oriented, which allows us to get detailed information on all exchanged messages and protocol events. We go beyond the mere observation of the good efficiency of both algorithms. We show that the rarest first algorithm guarantees close to ideal diversity of the pieces among peers. In particular, on our experiments, replacing the rarest first algorithm with source or network coding solutions cannot be justified. We also show that the choke algorithm in its latest version fosters reciprocation and is robust to free riders. In particular, the choke algorithm is fair and its replacement with a bit level tit-for-tat solution is not appropriate. Finally, we identify new areas of improvements for efficient peer-to-peer file replication protocols.
\end{abstract}

Categories and Subject Descriptors: C.2.2 [ComputerCommunication Networks]: Network Protocols; C.2.4 [Computer-Communication Networks]: Distributed Systems General Terms: Measurement, Algorithms, Performance Keywords: BitTorrent, choke algorithm, rarest first algorithm, peer-to-peer

\section{INTRODUCTION}

In a few years, peer-to-peer file sharing has become the most popular application in the Internet [16,17]. Efficient content localization and replication are the main reasons

(C) ACM, 2006. This is the author's version of the work. It is posted here by permission of ACM for your personal use. Not for redistribution. The definitive version was published in Proc. of IMC'06, October 25-27, 2006, Rio de Janeiro, Brazil. for this success. Whereas content localization has attracted considerable research interest in the last years $[7,12,23,25]$, content replication has started to be the subject of active research only recently. As an example, the most popular peer-to-peer file sharing networks [1] eDonkey2K, FastTrack, Gnutella, Overnet focus on content localization. The only widely used $[16,17,20]$ peer-to-peer file sharing application focusing on content replication is BitTorrent [8].

Yang et al. [26] studied the problem of efficient content replication in a peer-to-peer network. They showed that the capacity of the network to serve content grows exponentially with time in the case of a flash crowd, and that a key improvement on peer-to-peer file replication is to split the content into several pieces. Qiu et al. [22] proposed a refined model of BitTorrent and showed its high efficiency. In summary, these studies show that a peer-to-peer architecture for file replication is a major improvement compared to a client server architecture, whose capacity of service does not scale with the number of peers.

However, both studies assume global knowledge, which is not realistic. Indeed, they assume that each peer knows all the other peers. As a consequence, the results obtained with this assumption can be considered as the optimal case. In real implementations, there is no global knowledge. The challenge is then to design a peer-to-peer protocol that achieves a level of efficiency close to the one achieved in the case of global knowledge.

Piece and peer selection strategies are the two keys of efficient peer-to-peer content replication. Indeed, in a peerto-peer system, the content is split into several pieces, and each peer acts as a client and a server. Therefore, each peer can receive and give any piece to any other peer. An efficient piece selection strategy should guarantee that each peer can always find an interesting piece from any other peer. The rationale is to offer the largest choice of peers to the peer selection strategy. An efficient peer selection strategy should maximize the capacity of service of the system. In particular, it should employ selection criteria based, e.g., on upload and download capacity, and should not be biased by the lack of available pieces in some peers.

The rarest first algorithm is a piece selection strategy that consists of selecting the rarest pieces first. This simple strategy used by BitTorrent performs better than random piece selection strategies [5,9]. However, Gkantsidis et al. [11] argued based on simulations that the rarest first algorithm may lead to the scarcity of some pieces of content and proposed a solution based on network coding. Whereas this solution is elegant and has raised a lot of interest, it leads to several complex deployment issues such as security and computational cost. Other solutions based on source coding 
[18] have also been proposed to solve the claimed deficiencies of the rarest first algorithm.

The choke algorithm is the peer selection strategy of BitTorrent. This strategy is based on the reciprocation of upload and download speeds. Several studies $[5,10,13,15]$ discussed the fairness issues of the choke algorithm. In particular, they argued that the choke algorithm is unfair and favors free riders, i.e., peers that do not contribute. Solutions based on a bit level tit-for-tat have been proposed to address the choke algorithm's fairness problem.

In this paper, we perform an experimental evaluation of the piece and peer selection strategies as implemented in BitTorrent. Specifically, we have instrumented a client and run extensive experiments on several torrents with different characteristics in order to evaluate the properties of the rarest first and choke algorithms. While we have not examined all possible cases, we argue that we have covered a representative set of today real torrents.

Our main conclusions on real torrents are the following.

- The rarest first algorithm guarantees a high diversity of the pieces. In particular, it prevents the reappearance of rare pieces and of the last pieces problem.

- We have found that torrents in a startup phase can have low piece diversity. The duration of this phase depends only on the upload capacity of the source of the content. In particular, the rarest first algorithm is not responsible for the low piece diversity during this phase.

- The fairness achieved with a bit level tit-for-tat strategy is not appropriate in the context of peer-to-peer file replication. We have proposed two new fairness criteria in this context.

- The choke algorithm is fair, fosters reciprocation, and is robust to free riders in its latest version.

Our contribution is to go beyond the mere confirmation of the good performance of BitTorrent. We provide new insights into the role of peer and piece selection for efficient peer-to-peer file replication. We show for the first time that on real torrents, the efficiency of the rarest first and choke algorithms do no justify their replacement by more complex solutions. Also, we identify, based on our observations, new area of improvements: the replication of the first pieces and the speed of delivery of the first copy of the content. Finally, we propose two new fairness criteria in the context of peer-to-peer file replication and we present for the first time results on the new version of the choke algorithm that fixes fundamental fairness issues.

Our findings significantly differ from previous work $[5,10$, $11,13,15,18]$. There are three main reasons for this divergence. First, we target peer-to-peer file replication in the Internet. As a consequence, the peers are well connected without severe network bottlenecks. The problems identified in the literature with the rarest first algorithm are in the context of networks with connectivity problems or low capacity bottlenecks. Second, we evaluate for the first time the new version of the choke algorithm. The evaluation of the choke algorithm in the literature was performed on the old version. We show that the new version solves the problems identified on the old one. Finally, we perform an experimental evaluation on real torrents. Simulating peerto-peer protocols is hard and requires many simplifications. In particular, all the simulations of BitTorrent we are aware of consider that each peer only knows few other peers, i.e., each peer has a small peer set $[5,11]$. In the case of real torrents, the peer set size is much larger. The consequence is that BitTorrent builds a random graph, connecting the peers, that has a larger diameter in simulations than in real torrents. However, the diameter has a fundamental impact on the efficiency of the rarest first algorithm.

In this study, we show that in the specific context considered, i.e., Internet peer-to-peer file replication, the rarest first and choke algorithms are good enough. Even if we cannot extend our conclusions to other peer-to-peer contexts, we believe this paper sheds new light on a system that uses a large fraction of the Internet bandwidth.

The rest of the paper is organized as follows. We present the terminology used throughout this paper in section 2.1 Then, we give a short overview of the BitTorrent protocol in section 2.2 and a description of the rarest first and choke algorithms in section 2.3 We present our experimental methodology in section 3 and our detailed results in section 4 Related work is discussed in section [5] We conclude the paper with a discussion of the results in section 6

\section{BACKGROUND}

We introduce in this section the terminology used throughout this paper. Then, we give an overview of the BitTorrent protocol, and we present the rarest first and choke algorithms.

\subsection{Terminology}

The terminology used in the peer-to-peer community and in particular in the BitTorrent community is not standardized. For the sake of clarity, we define in this section the terms used throughout this paper.

- Pieces and Blocks Files transfered using BitTorrent are split in pieces, and each piece is split in blocks. Blocks are the transmission unit on the network, but the protocol only accounts for transfered pieces. In particular, partially received pieces cannot be served by a peer, only complete pieces can.

- Interested and Choked We say that peer $A$ is interested in peer $B$ when peer $B$ has pieces that peer $A$ does not have. Conversely, peer $A$ is not interested in peer $B$ when peer $B$ only has a subset of the pieces of peer $A$. We say that peer $A$ chokes peer $B$ when peer $A$ decides not to send data to peer $B$. Conversely, peer $A$ unchokes peer $B$ when peer $A$ decides to send data to peer $B$.

- Peer Set Each peer maintains a list of other peers it knows about. We call this list the peer set. The notion of peer set is also known as neighbor set.

- Local and Remote Peers We call local peer the peer with the instrumented BitTorrent client, and remote peers the peers that are in the peer set of the local peer.

- Active Peer Set A peer can only send data to a subset of its peer set. We call this subset the active peer set. The choke algorithm (described in section 2.3.2) determines the peers being part of the active peer set, i.e., which remote peers will be choked and unchoked. Only peers that are unchoked by the local peer and interested in the local peer are part of the active peer set. 
- Leecher and Seed A peer has two states: the leecher state, when it is downloading content, but does not have yet all the pieces; the seed state when the peer has all the pieces of the content. For short, we say that a peer is a leecher when it is in leecher state and a seed when it is in seed state.

- Initial Seed The initial seed is the peer that is the first source of the content.

- Rarest First Algorithm The rarest first algorithm is the piece selection strategy used in BitTorrent. We give a detailed description of this algorithm in section 2.3.1 The rarest first algorithm is also called the local rarest first algorithm.

- Choke Algorithm The choke algorithm is the peer selection strategy used in BitTorrent. We give a detailed description of this algorithm in section 2.3.2 The choke algorithm is also called the tit-for-tat algorithm, or tit-for-tat like algorithm.

- Rare and Available Pieces We call the pieces only present on the initial seed rare pieces, and we call the pieces already served at least once by the initial seed available pieces.

- Rarest Pieces and Rarest Pieces Set The rarest pieces are the pieces that have the least number of copies in the peer set. In the case the least replicated piece in the peer set has $m$ copies, then all the pieces with $m$ copies form the rarest pieces set. The rarest pieces can be rare pieces or available pieces, depending on the number of copies of the rarest pieces.

\subsection{BitTorrent Overview}

BitTorrent is a $\mathrm{P} 2 \mathrm{P}$ application that capitalizes on the bandwidth of peers to efficiently replicate contents on a large set of peers. A specificity of BitTorrent is the notion of torrent, which defines a session of transfer of a single content to a set of peers. Torrents are independent. In particular, participating in a torrent does not bring any benefit for the participation to another torrent. A torrent is alive as long as there is at least one copy of each piece in the torrent. Peers involved in a torrent cooperate to replicate the file among each other using swarming techniques [24]. In particular, the file is split in pieces of typically $256 \mathrm{kB}$, and each piece is split in blocks of $16 \mathrm{kB}$. Other piece sizes are possible.

A user joins an existing torrent by downloading a .torrent file usually from a Web server, which contains metainformation on the file to be downloaded, e.g., the piece size and the SHA-1 hash values of each piece, and the IP address of the so-called tracker of the torrent. The tracker is the only centralized component of BitTorrent, but it is not involved in the actual distribution of the file. It keeps track of the peers currently involved in the torrent and collects statistics on the torrent.

When joining a torrent, a new peer asks to the tracker a list of IP addresses of peers to build its initial peer set. This list typically consists of 50 peers chosen at random in the list of peers currently involved in the torrent. The initial peer set will be augmented by peers connecting directly to this new peer. Such peers are aware of the new peer by receiving its IP address from the tracker. Each peer reports its state to the tracker every 30 minutes in steady-state regime, or when disconnecting from the torrent, indicating each time the amount of bytes it has uploaded and downloaded since it joined the torrent. A torrent can thus be viewed as a collection of interconnected peer sets. If ever the peer set size of a peer falls below a predefined threshold, typically 20 peers, this peer will contact the tracker again to obtain a new list of IP addresses of peers. By default, the maximum peer set size is 80 . Moreover, a peer should not exceed a threshold of 40 initiated connections among the 80 at each time. As a consequence, the 40 remaining connections should be initiated by remote peers. This policy guarantees a good interconnection among the peer sets in the torrent.

Each peer knows the distribution of the pieces for each peer in its peer set. The consistency of this information is guaranteed by the exchange of messages [3]. The exchange of pieces among peers is governed by two core algorithms: the rarest first and the choke algorithms. These algorithms are further detailed in section 2.3

\subsection{BitTorrent Piece and Peer Selection Strategies}

We focus here on the two core algorithms of BitTorrent: the rarest first and choke algorithms. We do not give all the details of these algorithms, but explain the main ideas behind them.

\subsubsection{Rarest First Algorithm}

The rarest first algorithm works as follows. Each peer maintains a list of the number of copies of each piece in its peer set. It uses this information to define a rarest pieces set. Let $m$ be the number of copies of the rarest piece, then the index of each piece with $m$ copies in the peer set is added to the rarest pieces set. The rarest pieces set of a peer is updated each time a copy of a piece is added to or removed from its peer set. Each peer selects the next piece to download at random in its rarest pieces set.

The behavior of the rarest first algorithm can be modified by three additional policies. First, if a peer has downloaded strictly less than 4 pieces, it chooses randomly the next piece to be requested. This is called the random first policy. Once it has downloaded at least 4 pieces, it switches to the rarest first algorithm. The aim of the random first policy is to permit a peer to download its first pieces faster than with the rarest first policy, as it is important to have some pieces to reciprocate for the choke algorithm. Indeed, a piece chosen at random is likely to be more replicated than the rarest pieces, thus its download time will be on average shorter.

Second, BitTorrent also applies a strict priority policy, which is at the block level. When at least one block of a piece has been requested, the other blocks of the same piece are requested with the highest priority. The aim of the strict priority policy is to complete the download of a piece as fast as possible. As only complete pieces can be sent, it is important to minimize the number of partially received pieces.

Finally, the last policy is the end game mode [8]. This mode starts once a peer has requested all blocks, i.e., all blocks have either been already received or requested. While in this mode, the peer requests all blocks not yet received to all the peers in its peer set that have the corresponding blocks. Each time a block is received, it cancels the request for the received block to all the peers in its peer set that have the corresponding pending request. As a peer has a small buffer of pending requests, all blocks are effectively requested close to the end of the download. Therefore, the end game mode is used at the very end of the download, thus it has little impact on the overall performance. 


\subsubsection{Choke Algorithm}

The choke algorithm was introduced to guarantee a reasonable level of upload and download reciprocation. As a consequence, free riders, i.e., peers that never upload, should be penalized. For the sake of clarity, we describe without loss of generality the choke algorithm from the point of view of the local peer. In this section, interested always means interested in the local peer, and choked always means choked by the local peer.

The choke algorithm differs in leecher and seed states. We describe first the choke algorithm in leecher state. At most 4 remote peers can be unchoked and interested at the same time. Peers are unchoked using the following policy.

1. Every 10 seconds, the interested remote peers are ordered according to their download rate to the local peer and the 3 fastest peers are unchoked.

2. Every 30 seconds, one additional interested remote peer is unchoked at random. We call this random unchoke the optimistic unchoke.

In the following, we call the three peers unchoked in step 1 the regular unchoked (RU) peers, and the peer unchoked in step 2 the optimistic unchoked (OU) peer. The optimistic unchoke peer selection has two purposes. It allows to evaluate the download capacity of new peers in the peer set, and it allows to bootstrap new peers that do not have any piece to share by giving them their first piece.

We describe now the choke algorithm in seed state. In previous versions of the BitTorrent protocol, the choke algorithm was the same in leecher state and in seed state except that in seed state the ordering performed in step 1 was based on upload rates from the local peer. With this algorithm, peers with a high download rate are favored independently of their contribution to the torrent.

Starting with version 4.0.0, the mainline client [2] introduced an entirely new algorithm in seed state. We are not aware of any documentation on this new algorithm, nor of any implementation of it apart from the mainline client.

We describe this new algorithm in seed state in the following. At most 4 remote peers can be unchoked and interested at the same time. Peers are unchoked using the following policy.

1. Every 10 seconds, the unchoked and interested remote peers are ordered according to the time they were last unchoked, most recently unchoked peers first.

2. For two consecutive periods of 10 seconds, the 3 first peers are kept unchoked and an additional $4^{\text {th }}$ peer that is choked and interested is selected at random and unchoked.

3. For the third period of 10 seconds, the 4 first peers are kept unchoked.

In the following, we call the three or four peers that are kept unchoked according to the time they were last unchoked the seed kept unchoked (SKU) peers, and the unchoked peer selected at random the seed random unchoked (SRU) peer. With this new algorithm, peers are no longer unchoked according to their upload rate from the local peer, but according to the time of their last unchoke. As a consequence, the peers in the active peer set are changed regularly, each new SRU peer taking an unchoke slot off the oldest SKU peer.

We show in section 4.2.1 why the new choke algorithm in seed state is fundamental to the fairness of the choke algorithm.

\section{EXPERIMENTAL METHODOLOGY}

In order to evaluate experimentally the rarest first and choke algorithms on real torrents, we have instrumented a BitTorrent client and connected this client to live torrents with different characteristics. The experiments were performed one at a time in order to avoid a possible bias due to overlapping experiments. We have instrumented a single client and we make no assumption on the other clients connected to the same torrent. As we only considered real torrents, we captured a large variety of client configuration, connectivity, and behavior. In the following, we give details on how we conducted the experiments.

\subsection{Choice of the Monitored BitTorrent Client}

Several BitTorrent clients are available. The first BitTorrent client has been developed by Bram Cohen, the inventor of the protocol. This client is open source and is called mainline [2]. As there is no well maintained and official specification of the BitTorrent protocol, the mainline client is considered as reference for the BitTorrent protocol. It should be noted that, up to now, each improvement of Bram Cohen to the BitTorrent protocol has been replicated to the most popular other clients.

The other clients differ from the mainline client by a more sophisticated interface with a nice look and feel, realtime statistics, many configuration options, experimental extensions to the protocol, etc.

Since our goal is to evaluate the basic BitTorrent protocol, we have decided to restrict ourselves to the mainline client. This client is very popular as it is the second most downloaded BitTorrent client at SourceForge with more than 52 million downloads. We instrumented the version 4.0.2 of the mainline client released at the end of May $2005^{1}$. This version of the instrumented mainline client implements the new choke algorithm in seed state (see section [2.3.2].

\subsection{Choice of the Torrents}

The aim of this work is to understand how the rarest first and choke algorithms behave on real torrents. It is not intended to provide an exhaustive study on the characteristics of today's torrents. For this reason, we have selected torrents based on: their proportion of seeds to leechers, the absolute number of seeds and leechers, and the content size. The torrents monitored in this study were found on popular sites ${ }^{2}$. We considered copyrighted and free contents, which are TV shows, movies, cartoons, music albums, live concert recordings, and softwares. Each experiment lasted for 8 hours in order to make sure that each client became a seed and to have a representative trace in seed state. We performed all the experiments between June 2005 and May 2006.

We give the characteristic of each torrent in Table 1 The number of seeds and leechers is given at the beginning of the experiment. Therefore, these numbers can be very different at the end of the experiment. We see that there is a large variety of torrents: torrents with few seeds and few leechers, torrents with few seeds and a large number of leech-

\footnotetext{
${ }^{1}$ The latest stable branch of development is 4.20.x. In this branch, there is no new functionality to the core protocol, but a new tracker-less functionality and some improvements to the client. As the evaluation of the tracker functionality was outside the scope of this study we focused on version 4.0.2.

${ }^{2}$ www.legaltorrents.com, bt.etree.org, fedora.redhat.com, www.mininova.org, isohunt.com.
} 
Table 1: Torrent characteristics. Column 1 (ID): torrent ID, column 2 (\# of $\mathbf{S}$ ): number of seeds at the beginning of the experiment, column 3 (\# of L): number of leechers at the beginning of the experiment, column 4 (Ratio $\frac{S}{L}$ ): ratio (number of seeds)/(number of leechers), column 5 (Max. PS): maximum peer set size in leecher state, column 6 (Size): size of the content in MB.

\begin{tabular}{|c|c|c|c|c|c|}
\hline ID & \# of S & \# of L & Ratio $\frac{S}{L}$ & Max. PS & Size \\
\hline 1 & 0 & 66 & 0 & 60 & 700 \\
\hline 2 & 1 & 2 & 0.5 & 3 & 580 \\
\hline 3 & 1 & 29 & 0.034 & 34 & 350 \\
\hline 4 & 1 & 40 & 0.025 & 75 & 800 \\
\hline 5 & 1 & 50 & 0.02 & 60 & 1419 \\
\hline 6 & 1 & 130 & 0.0078 & 80 & 820 \\
\hline 7 & 1 & 713 & 0.0014 & 80 & 700 \\
\hline 8 & 1 & 861 & 0.0012 & 80 & 3000 \\
\hline 9 & 1 & 1055 & 0.00095 & 80 & 2000 \\
\hline 10 & 1 & 1207 & 0.00083 & 80 & 348 \\
\hline 11 & 1 & 1411 & 0.00071 & 80 & 710 \\
\hline 12 & 3 & 612 & 0.0049 & 80 & 1413 \\
\hline 13 & 9 & 30 & 0.3 & 35 & 350 \\
\hline 14 & 20 & 126 & 0.16 & 80 & 184 \\
\hline 15 & 30 & 230 & 0.13 & 80 & 820 \\
\hline 16 & 50 & 18 & 2.8 & 40 & 600 \\
\hline 17 & 102 & 342 & 0.3 & 80 & 200 \\
\hline 18 & 115 & 19 & 6 & 55 & 430 \\
\hline 19 & 160 & 5 & 32 & 17 & 6 \\
\hline 20 & 177 & 4657 & 0.038 & 80 & 2000 \\
\hline 21 & 462 & 180 & 2.6 & 80 & 2600 \\
\hline 22 & 514 & 1703 & 0.3 & 80 & 349 \\
\hline 23 & 1197 & 4151 & 0.29 & 80 & 349 \\
\hline 24 & 3697 & 7341 & 0.5 & 80 & 349 \\
\hline 25 & 11641 & 5418 & 2.1 & 80 & 350 \\
\hline 26 & 12612 & 7052 & 1.8 & 80 & 140 \\
\hline
\end{tabular}

ers, torrents with a large number of seeds and few leechers, and torrents with a large number of seeds and leechers. We discuss in section 3.5 .2 the limitations in the choice of the torrents considered.

\subsection{Experimental Setup}

We performed a complete instrumentation of the mainline client. The instrumentation consists of: a log of each BitTorrent message sent or received with the detailed content of the message, a log of each state change in the choke algorithm, a log of the rate estimation used by the choke algorithm, and a log of important events (end game mode, seed state).

As monitored client, we use the mainline client with all the default parameters for all our experimentations. It is outside of the scope of this study to evaluate the impact of each BitTorrent parameter. The main default parameters for the monitored client are: the maximum upload rate (default to $20 \mathrm{kB} / \mathrm{s}$ ), the minimum number of peers in the peer set before requesting more peers to the tracker (default to 20), the maximum number of connections the local peer can initiate (default to 40), the maximum number of peers in the peer set (default to 80), the number of peers in the active peer set including the optimistic unchoke (default to 4 ), the block size (default to $2^{14}$ Bytes), the number of pieces downloaded before switching from random to rarest first piece selection (default to 4 ).

We did all our experimentations on a machine connected to a high speed backbone. However, the upload capacity is limited by default by the client to $20 \mathrm{kB} / \mathrm{s}$. There is no limit to the download capacity. We obtained effective maximum download speed ranging from $20 \mathrm{kB} / \mathrm{s}$ up to 1500 $\mathrm{kB} / \mathrm{s}$ depending on the experiments. We ran between 1 and 3 experiments on the 26 different torrents given in Table 1 and performed a detailed analysis of each of these traces. The results given in this paper are for a single run for each torrent. Multiple runs on some torrents were used in a calibration phase as explained in section 3.5.1

Finally, whereas we have control over the monitored mainline client, we do not control any other client in a torrent. In particular, all peers in the peer set of the local peer are real live peers.

\subsection{Peer Identification}

In our experiments, we uniquely identify a peer by its IP address and peer ID. The peer ID, which is 20 bytes, is a string composed of the client ID and a randomly generated string. This random string is regenerated each time the client is restarted. The client ID is a string composed of the client name and version number, e.g., M4-0-2 for the mainline client in version 4.0.2. We are aware of around 20 different BitTorrent clients, each client existing in several different versions. When in a given experiment, we see several peer IDs corresponding to the same IP address ${ }^{3}$, we compare the client ID of the different peer IDs. In the case the client ID is the same for all the peer IDs on a same IP address, we deem that this is the same peer. We cannot rely on the peer ID comparison, as the random string is regenerated each time a client crashes or restarts. The pair (IP, client ID) does not guarantee that each peer can be uniquely identified, because several peers beyond a NAT can use the same client in the same version. However, considering the large number of client IDs, it is common in our experiments to observe 15 different client IDs, the probability to have several different clients beyond a NAT with the same client ID is reasonably low for our purposes. Moreover, unlike what was reported by Bhagwan et al. [4] for the Overnet file sharing network, we did not see any problem of peer identification due to NATs. In fact, BitTorrent has an option, activated by default, to prevent accepting multiple concurrent incoming connections from the same IP address. The idea is to prevent peers to increase their share of the torrent, by opening multiple clients from the same machine. Therefore, even if we found in our traces different peers with the same IP address at different moments in time, two different peers with the same IP address cannot be connected to the local peer during overlapping periods.

\subsection{Limitations and Interpretation of the Re- sults}

In this section we discuss the two main limitations of this work, namely the single client instrumentation and the limited set of monitored torrents. We also discuss why, despite these limitations, we believe our conclusions hold for a broader range of scenarios than the ones presented.

\subsubsection{Single Client Instrumentation}

We have chosen for this study to focus on the behavior of a single client in a real torrent. Whereas it may be argued that a larger number of instrumented peers would have given a better understanding of the torrents, we made the

\footnotetext{
${ }^{3}$ Between $0 \%$ and $26 \%$ of the IP addresses, depending on the experiments, are associated in our traces to more than one peer ID. The mean is around $9 \%$.
} 
decision to be as unobtrusive as possible. Increasing the number of instrumented clients would have required to either control those clients ourselves, or to ask some peers to use our instrumented client. In both cases, the choice of the instrumented peer set would have been biased, and the behavior of the torrent impacted. Instead, our decision was to understand how a new peer (our instrumented peer) joining a real torrent behaves.

Moreover, monitoring a single client does not adversely impact the generality of our findings for the following reasons. First, a torrent is a random graph of interconnected peers. For this reason, with a large peer set of 80 , each peer should have a view of the torrent as representative as any other peer. Even if each peer will see variations due to the random choice of the population in its peer set, the big picture will remain the same. Second, in order to make sure that there is no unforeseen bias due to the single client instrumentation, we have monitored several torrents with three different peers, each peer with a different IP address. These experiments were performed during a calibration phase, and are not presented here due to space limitation. Whereas the download speed of the peers may significantly vary, e.g., due to very fast seeds that may of may not be present in the peer set of a monitored client, we did not observe any other significant difference among the clients that may challenge the generality of our findings.

\subsubsection{Limited Torrent Set}

We have considered for this study 26 different torrents. Whereas it is a large number of torrents, it is not large enough to be exhaustive or to be representative of all the torrents that can be found in the Internet. However, our intent is to evaluate the behavior of the rarest first and choke algorithm in a variety of situations. The choice of the torrents considered in this study was targeted to provide a challenging environment to the rarest first and choke algorithms. For instance, torrents with no seed (torrent 1) or with only one seed and a large number of leechers (e.g., torrent 711) were specifically chosen to evaluate how the rarest first algorithm behaves in the context of pieces scarcity. Torrents with a large number of peers were selected to evaluate how the choke algorithm behaves when the torrent is large enough to favor free riders.

We have around half of the presented torrents with no or few seeds, as this is a challenging situation for a peer-topeer protocol. However, it can be argued that the largest presented torrent with a single seed has a small number of leechers (1441 leechers at the beginning of the experiment for torrent 11). Indeed, the target of a peer-to-peer protocol is to distribute content to millions of peers. But, a peerto-peer protocol capitalizes on the bandwidth of each peer. Thus, it is not possible to scale to millions of peers without a significant proportion of seeds. If we take the same proportion of seeds and leechers as the one of torrent 11, only 710 seeds are enough to scale to one million of peers. Also, a torrent with a ratio $\frac{\text { number of seeds }}{\text { number of leechers lower than } 10^{-3} \text { is }}$ enough to stress a piece selection strategy based on a local view of only 80 peers.

Finally, in such an experimental study it is not possible to reproduce an experiment, and thus to gain statistical information because each experiment depends on the behavior of peers, the number of seeds and leechers in the torrent, and the subset of peers randomly returned by the tracker. However, studying the dynamics of the protocol is as important as studying its statistical properties. As we considered torrents with different characteristics and observed a consistent behavior on these torrents, we believe our findings to be representative of the rarest first and choke algorithms behavior.

\section{EXPERIMENTAL RESULTS}

We present in this section the results of our experiments. In a first part, we discuss the results with a focus on the rarest first algorithms. Then, in a second part, we discuss the results with a focus on the choke algorithm.

\subsection{Rarest First Algorithm}

The aim of a piece selection strategy is to guarantee that each peer is always interested in any other peer. The rational is that each time the peer selection strategy unchokes a peer, this peer must be interested in the unchoking peer. This way, the peer selection strategy can reach the optimal system capacity (but, designing such an optimal peer selection strategy is a hard task). Therefore, the piece selection strategy is fundamental to reach good system capacity.

However, the efficiency of the piece selection strategy cannot be measured in terms of system capacity, because the system capacity is the result of both the piece and peer selection strategies. A good way to evaluate the efficiency of the piece selection strategy is to measure the entropy of the torrent, i.e., the repartition of pieces among peers.

There is no simple way to directly measure the entropy of a torrent. For this reason, we characterize the entropy with the peer availability. We define the peer availability of peer $x$ according to peer $y$ as the ratio of the time peer $y$ is interested (see section 2.1) in peer $x$ over the time peer $x$ is in the peer set of peer $y$. If peer $x$ is always available for peer $y$, then the peer availability is equal to one. In the following, we characterize the entropy of a torrent with the availability of the peers in this torrent. For the sake of clarity, we will simply refer to the notion of entropy.

We say that there is ideal entropy in a torrent when each leecher ${ }^{4}$ is always interested in any other leecher. We do not claim that ideal entropy can be always achieved, but it should be the objective of any efficient piece selection strategy.

We evaluated the rarest first algorithm on a representative set of real torrents. We showed that the rarest first algorithm achieves a close to ideal entropy, and that its replacement by more complex solutions cannot be justified. Then, we evaluated the dynamics of the rarest first algorithm to understand the reasons for this good entropy. Finally, we focused on a specific problem called the last pieces problem, which is presented $[11,18]$ as a major weakness of the rarest first strategy. We showed that the last pieces problem is overestimated. In contrast, we identified a first blocks problem, which is a major area of improvement for BitTorrent.

\subsubsection{Entropy Characterization}

The major finding of this section is that the rarest first algorithm achieves a close to ideal entropy for real torrents. We remind that ideal entropy is achieved when each leecher is always interested in any other leecher. As we do not have global knowledge of the torrent, we characterize the entropy from the point of view of the local peer with two ratios. For each remote peer we compute:

\footnotetext{
${ }^{4}$ Only the case of leechers is relevant for the entropy characterization, as seeds are always interesting for leechers and never interested in leechers.
} 


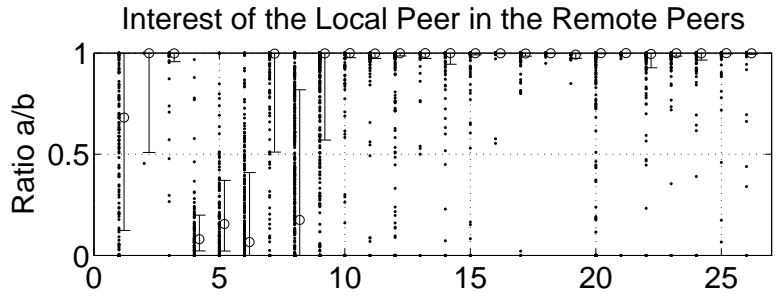

Interest of the Remote Peers in the Local Peer

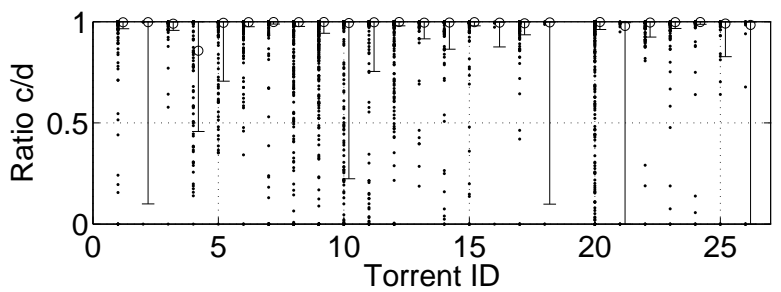

Figure 1: Entropy characterization. Top graph: For each remote leecher peer for a given torrent, a dot represents the ratio $\frac{a}{b}$ where $a$ is the time the local peer in leecher state is interested in this remote peer and $b$ is the time this remote peer spent in the peer set when the local peer is in leecher state. Bottom graph: For each remote leecher peer for a given torrent, a dot represents the ratio $\frac{c}{d}$ where $c$ is the time this remote peer is interested in the local peer in leecher state and $d$ is the time this remote peer spent in the peer set when the local peer is in leecher state. For both graphs: Each vertical solid lines represent the $20^{\text {th }}$ percentile (bottom of the line), the median (identified with a circle), and the $80^{\text {th }}$ percentile (top of the line) of the ratios for a given torrent.

- the ratio $\frac{a}{b}$ where $a$ is the time the local peer in leecher state is interested in this remote peer and $b$ is the time this remote peer spent in the peer set when the local peer is in leecher state;

- the ratio $\frac{c}{d}$ where $c$ is the time this remote peer is interested in the local peer in leecher state and $d$ is the time this remote peer spent in the peer set when the local peer is in leecher state.

In the case of ideal entropy the above ratios should be one. Fig. 1 gives a characterization of the entropy for the torrents considered in this study.

For most of our torrents, we see in Fig. 1 that the ratios are close to 1 , thus a close to ideal entropy. For the top graph, $70 \%$ of the torrents have the $20^{\text {th }}$ percentile close to one, and $80 \%$ have the median close to one. For the bottom graph, $70 \%$ of the torrents have a $20^{\text {th }}$ percentile close to one, and $90 \%$ of the torrents have the median close to one. We discuss below the case of the torrents with low entropy.

First, we discuss why the local peer is often not interested in the remote peers for torrents $1,2,4,5,6,7,8$, and 9 (see Fig. 1 top graph). These torrents have low entropy because they are in a startup phase. This means that the initial seed has not yet served all the pieces of the content. We remind that the pieces only present on the initial seed are the rare pieces, and that the pieces already served at least once by the initial seed are the available pieces (see section 2.1). The reason for the low observed entropy is that during a torrent startup, available pieces are replicated with an exponential capacity of service [26], but rare pieces are served by the initial seed at a constant rate. Thus, available pieces are replicated faster than rare pieces. This leads to two problems. First, the probability of having peers in a peer set with the same subset of pieces is higher during the torrent startup than when there is no rare piece in the torrent. Second, when there is no rare piece, a peer with all the available pieces becomes a seed. But, when there are rare pieces, a peer with all the available pieces remains a leecher because it does not have the rare pieces. However, these leechers cannot be interested in any other peer as they have all the available pieces at this point of time, but they stay in the peer set of the local peer. Thus a low ratio for these leechers in Fig. 11 In conclusion, the low entropy we observed is not due to a deficiency of the rarest first algorithm, but to the startup phase of the torrent whose duration depends only on the upload capacity of the initial seed. We discuss further this point in section 4.1.2.1

Now, we discuss why the remote peers are often not interested in the local peer for torrents $2,4,10,18,19,21$, and 26 (see Fig. 1 bottom graph). No dot is displayed for torrent 19 because due to the small number of leechers in this torrent, the local peer in leecher state had no leecher in its peer set. Five torrents have a $20^{\text {th }}$ percentile close to 0 . The percentile for four of these torrents is computed on a small number of ratios: 3, 8, 12, and 15 for torrents 2, 18,21 and 26 respectively. Therefore, the $20^{\text {th }}$ percentile is not representative as it is not computed on a set large enough. Additionally, the reason for the low $20^{\text {th }}$ percentile is peers with a ratio of 0 . We identified two reasons for a ratio of 0 . First, some peers join the peer set with almost all pieces. They are therefore unlikely to be interested in the local peer. Second, some peers with no or few pieces never sent an interested message to the local peer. This can be explained by a client behavior changed with a plugin or an option activation. The super seeding option [3] available in several BitTorrent clients has this effect. In conclusion, the low entropy of some peers is either a measurement artifact due to modified or misbehaving clients, or the result of the inability of the rarest first algorithm to reach ideal entropy in some extreme cases.

We have seen that peers that join the torrent with almost all pieces may not be interested in the local peer. In this scenario, the rarest first algorithm does not guarantee ideal entropy. However, we argue that this case does not justify the replacement of the rarest first algorithm for two reasons. First, this case appears rarely and does not significantly impact the overall entropy of the torrent. Second, the peers with low entropy are peers that join the peer set with only a few missing pieces. In the case of torrent startup, it is not clear whether a solution based, for instance, on source or network coding would have proposed interesting pieces to such peers. Indeed, when content is split into $k$ pieces, there is no solution based on coding that can reconstruct the content in less than $k$ pieces. For this reason, when the initial seed has not yet sent at least one copy of each piece, there is no way to reconstruct the content, so no way to have interesting pieces for all the peers.

An important question is how rarest first compares with network coding in the presented scenarios. As there is no client based on network coding that is as popular as BitTorrent, it is not possible to evaluate both solutions on the same torrents. However, based on the theoretical network coding results, we discuss the respective merits of rarest first and network coding in section 4.1.4

For the computation of the ratios on Fig. 1] we did not consider peers that spent less than 10 seconds in the peer 
set. Our motivation was to evaluate the entropy of pieces in a torrent. However, due to several misbehaving clients, there is a permanent noise created by peers that join and leave the peer set frequently. Such peers stay typically less than a few seconds in the peer set, and they do not take part in any active upload or download. Therefore, these misbehaving peers adversely bias our entropy characterization. Filtering all peers that stay less than 10 seconds remove the bias.

In summary, we have seen that the rarest first algorithm enforces a close to ideal entropy for the presented torrents. We have identified torrents with low entropy and shown that the rarest first algorithm is not responsible for this low entropy. We have also identified rare cases where the rarest first algorithm does not perform optimally, but we have explained that these cases do not justify a replacement with a more complex solution. In the following, we evaluate how the rarest first piece selection strategy achieves high entropy.

\subsubsection{Rarest First Algorithm Dynamics}

We classify a torrent in two states: the transient state and the steady state $^{5}$. In transient state, there is only one seed in the torrent. In particular, there are some pieces that are rare, i.e., present only at the seed. This state corresponds to the beginning of the torrent, when the initial seed has not yet uploaded all the pieces of the content. All torrents with low entropy (Fig. 1 top graph) are in transient state. A good piece replication algorithm should minimize the time spent in the transient state because low entropy may adversely impact the service capacity of a torrent by biasing the peer selection strategy. In steady state, there is no rare piece, and the piece replication strategy should prevent the torrent to enter again a transient state. All torrents with high entropy are in steady state.

In the following, we evaluate how the rarest first algorithm performs in transient and steady state. We show that the low entropy of torrents experienced in transient state is due to the limited upload capacity of the initial seed, and that the rarest first algorithm minimizes the time spent in this state. We also show that the rarest first algorithm is efficient at keeping a torrent in steady state, thus guaranteeing a high entropy.

\subsubsection{Transient State.}

In order to understand the dynamics of the rarest first algorithm in transient state, we focus on torrent 8 . This torrent consisted of 1 seed and 861 leechers at the beginning of the experiment. The file distributed in this torrent is split in 863 pieces. We run this experiment during 58991 seconds, but in the following we only discuss the results for the first 29959 seconds when the local peer is in leecher state.

Torrent 8 is in transient state for most of the experiment. As we don't have global knowledge of the torrent, we do not have a direct observation of the transient state. However, there are several evidences of this state. Indeed, Fig. 2] shows that there are missing pieces during the experiment in the local peer set, as the minimum curve (dashed line) is at zero. Moreover, we probed the tracker to get statistics on the number of seeds and leechers during this experiment. We found that this torrent had only one seed for the duration of the experiment.

We see in Fig. 1 top graph, that torrent 8 has low entropy. This low entropy is due to the limited upload capacity of the initial seed. Indeed, when a torrent is in transient state,

\footnotetext{
${ }^{5}$ Our definition of transient and steady state differs from the one given by Yang et al. [26].
}

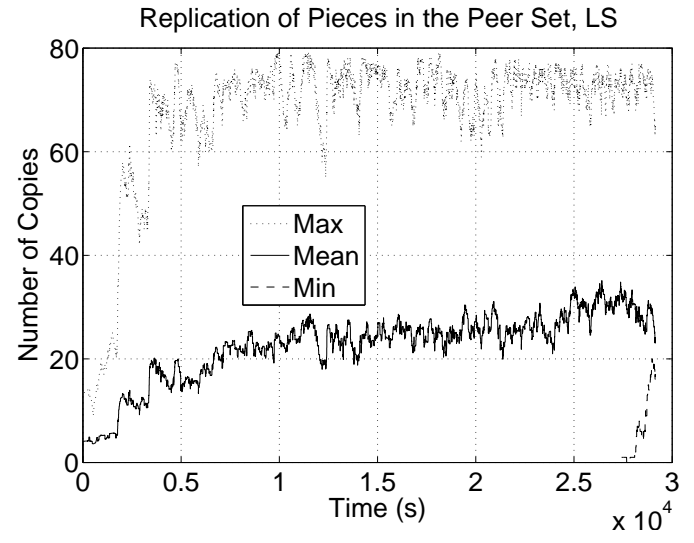

Figure 2: Evolution of the number of copies of pieces in the peer set with time for torrent 8 in leecher state. Legend: The dotted line represents the number of copies of the most replicated piece in the peer set at each instant. The solid line represents the mean number of copies over all the pieces in the peer set at each instant. The dashed line represents the number of copies of the least replicated piece in the peer set at each instant.

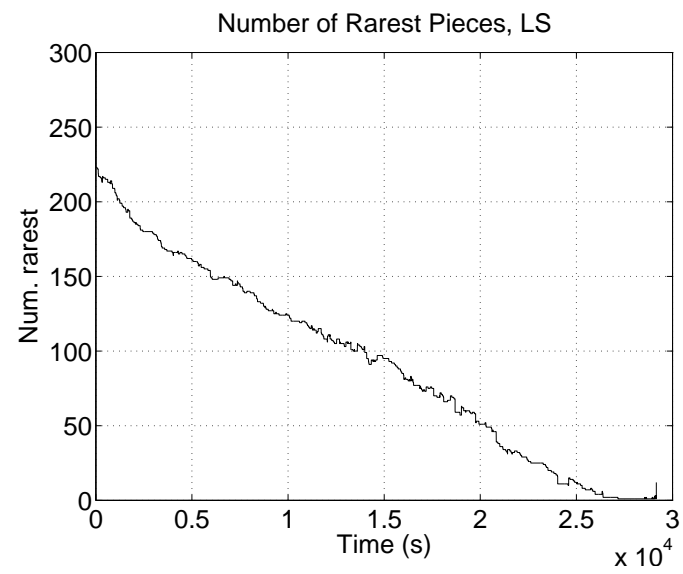

Figure 3: Evolution of the number of rarest pieces in the peer set for torrent 8 in leecher state. The rarest pieces set is formed by the pieces that are equally the rarest, i.e., the pieces that have the least number of copies in the peer set.

available pieces are replicated with an exponential capacity of service [26], but rare pieces are served by the initial seed at a constant rate. This is confirmed by Fig. 3 that shows the number of rarest pieces, i.e., the set size of the pieces that are equally rarest. We see that the number of rarest pieces decreases linearly with time. As the size of each piece in this torrent is $4 \mathrm{MB}$, a rapid calculation shows that the rarest pieces are duplicated in the peer set at a constant rate close to $36 \mathrm{kB} / \mathrm{s}$. We do not have a direct proof that this rate is the one of the initial seed, because we do not have global knowledge of the torrent. However, the torrent is in its startup phase and most of the pieces are only available on the initial seed. Indeed, Fig. 2 shows that there are missing pieces in the peer set, thus the rarest pieces presented in Fig. 3 are missing pieces in the peer set. Therefore, only the initial seed can serve the missing pieces shown in Fig. 3 In conclusion, the upload capacity of the initial seed is the 
bottleneck for the replication of the rare pieces, and the time spent in transient state only depends on the upload capacity of the initial seed.

The rarest first algorithm attempts to minimize the time spent in transient state and replicates fast available pieces. Indeed, leechers download first the rare pieces. As the rare pieces are only present on the initial seed, the upload capacity of the initial seed will be fully utilized and no or few duplicate rare pieces will be served by the initial seed. Once served by the initial seed, a rare piece becomes available and is served in the torrent with an increasing capacity of service. As rare pieces are served at a constant rate, most of the capacity of service of the torrent is used to replicate the available pieces on leechers. Indeed, Fig. 2 shows that once a piece is served by the initial seed, the rarest first algorithm will start to replicate it fast as shown by the continuous increase in the mean number of copies over all the peers, and by the number of copies of the most replicated piece (dotted line) that is always close to the maximum peer set size of 80.

In summary, the low entropy observed for some torrents is due to the transient phase. The duration of this phase cannot be shorter than the time for the initial seed to send one copy of each piece, which is constrained by the upload capacity of the initial seed. Thus, the time spent in this phase cannot be shorten further by the piece replication strategy. The rarest first algorithm minimizes the time spent in transient state. Once a piece is served by the initial seed, the rarest first algorithm replicates it fast. Therefore, a replacement of the rarest first algorithm by another algorithm cannot be justified based on the real torrents we have monitored in transient state.

\subsubsection{Steady State.}

In order to understand the dynamics of the rarest first algorithm in steady state, we focus on torrent 7 . This torrent consisted of 1 seed and 713 leechers at the beginning of the experiment. We have seen on Fig. 1 that torrent 7 has a high entropy. Fig. 4 shows that the least replicated piece (min curve) has always more than 1 copy in the peer set. Thus, torrent 7 is in steady state.

In the following, we present the dynamics of the rarest first algorithm in steady state, and explain how this algorithm prevents the torrent to return in transient state. Fig. 4] shows that the mean number of copies remains well bounded over time by the number of copies of the most and least replicated pieces. The variation observed in the number of copies are explained by the variation of the peer set size, see Fig. 5 The decrease in the number of copies 9051 seconds after the beginning of the experiment corresponds to the local peer switching to seed state. Indeed, when a leecher becomes a seed, it closes its connections to all the seeds.

The rarest first algorithm does a very good job at increasing the number of copies of the rarest pieces. Fig. 4 shows that the number of copies of the least replicated piece (min curve) closely follows the mean, but does not significantly get closer. However, we see in Fig. 6] that the number of rarest pieces, i.e., the set size of the pieces that are equally rarest, follow a sawtooth behavior. Each peer joining or leaving the peer set can alter the set of rarest pieces. But, as soon as a new set of pieces becomes rarest, the rarest first algorithm quickly duplicates them as shown by a consistent drop in the number of rarest pieces in Fig[6] Finally, we never observed in any of our torrents a steady state followed by a transient state.

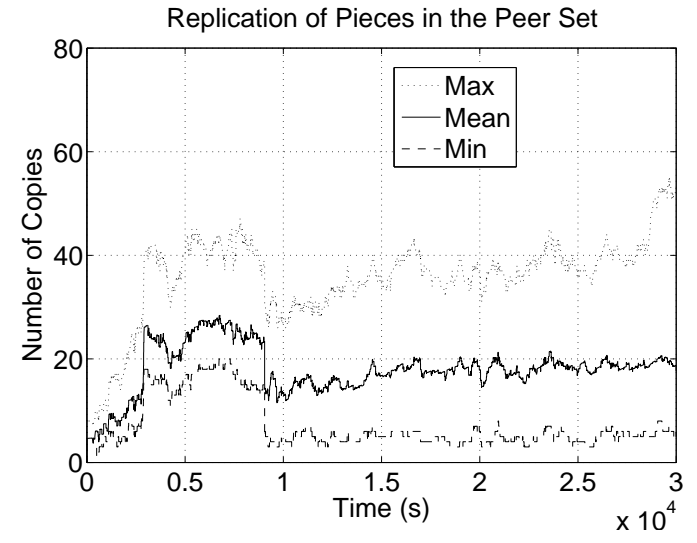

Figure 4: Evolution of the number of copies of pieces in the peer set with time for torrent 7. Legend: The dotted line represents the number of copies of the most replicated piece in the peer set at each instant. The solid line represents the mean number of copies over all the pieces in the peer set at each instant. The dashed line represents the number of copies of the least replicated piece in the peer set at each instant.

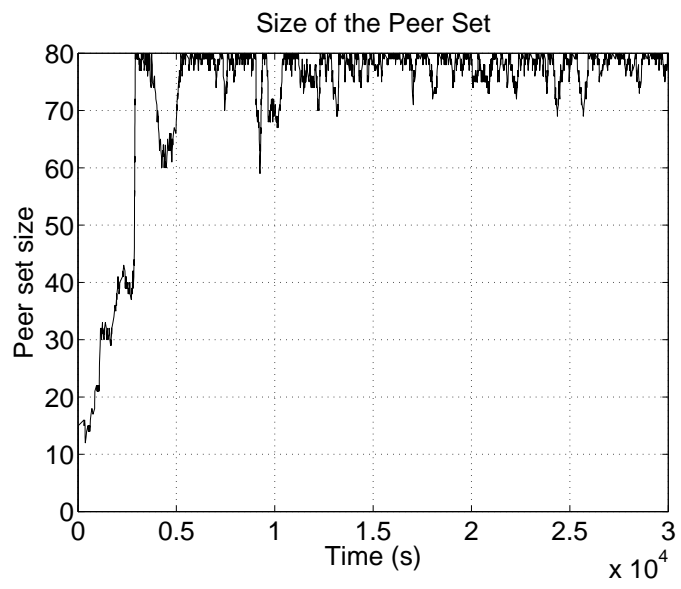

Figure 5: Evolution of the peer set size for torrent 7.

In summary, the rarest first algorithm in steady state ensures a good replication of the pieces in real torrents. It also replicates fast the rarest pieces in order to prevent the reappearance of a transient state. We conclude that on real torrents in steady state, the rarest first algorithm is enough to guarantee a high entropy.

\subsubsection{Last Pieces Problem}

We say that there is a last pieces ${ }^{6}$ problem when the download speed suffers a significant slow down for the last pieces. This problem is due to some pieces replicated on few overloaded peers, i.e., peers that receive more requests than they can serve. This problem is detected by a peer only at the end of the content download. Indeed, a peer always seeks for fast peers to download from. Thus, it is likely that if some pieces are available on only few overloaded peers, these peers

\footnotetext{
${ }^{6}$ This problem is usually referenced as the last piece (singular) problem. However, there is no reason why this problem affects only a single piece.
} 


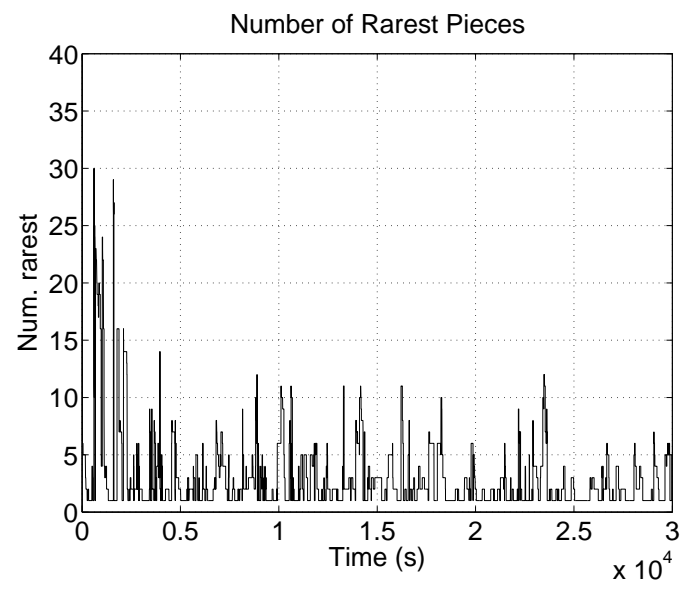

Figure 6: Evolution of the number of rarest pieces in the peer set for torrent 7 . The rarest pieces set is formed by the pieces that are equally the rarest, i.e., the pieces that have the least number of copies in the peer set.

will be chosen only at the end of the content download when there is no other pieces to download.

Due to space limitation, we just give our main conclusions. For a detailed discussion on the last pieces problem, the interested reader may refer to [19].

We never observed a last pieces problem on torrents in steady state. However, we observed this problem on a few torrents in transient state. We found that this problem is inherent to the transient state of the torrent, and is not due to the rarest first algorithm. Moreover, the rarest first algorithm is efficient at mitigating this problem by replicating fast rare pieces once they become available.

It is important to study the piece interarrival time, because partially received pieces cannot be retransmitted by a BitTorrent client, only complete pieces can. However, pieces are split into blocks, which are the BitTorrent unit of data transfer. For this reason, we have also evaluated the block interarrival time. We identified a first blocks problem. This first blocks problem results in a slow startup of the torrent, which is an area of improvement for BitTorrent.

In conclusion, the last pieces problem is overstated, but the first blocks problem is underestimated and a possibility of performance improvement.

\subsubsection{Discussion on Rarest First and Network Cod- ing}

We have seen that rarest first is an efficient piece selection strategy on the presented torrents. We have also shown that the claimed deficiencies of rarest first cannot be identified in our experiments, or are the results of a misunderstanding of the reason of piece scarcity for torrents in transient state.

However, this paper is not a case against solutions based on source or network coding. Network coding enables a piece selection strategy that is close to optimal in all cases, which is not the case of rarest first. Indeed, in specific contexts like small outdegree constraint, or poor network connectivity between cluster of peers, rarest first will perform poorly. In this study, we show that on real torrents in the Internet, which have a large peer set of 80 and do not suffer from connectivity problems, rarest first performs very well.

In fact, rarest first is close to a solution based on network coding in the presented torrents. We consider two cases to make the comparison: the steady and transient states. In steady state, we have seen in section 4.1.2.2 that the entropy of the presented torrents is close to one with rarest first. An entropy close to one means that each peer is interested in each other peer in its peer set most of the time. As this is close to the target of an ideal piece selection strategy, we see that in steady state, the possibility of improvement for any piece selection strategy in not significant compared to rarest first. For this reason, we argue that a replacement of rarest first cannot be justified in the studied context. In transient state, a solution based on network coding will enable the initial seed to send one entire copy of the content faster than in the case of rarest first that may suffer from duplicate pieces. The problem with rarest first is that the number of duplicate pieces will depends on the peer selection strategy. Indeed, if the initial seed chooses the same set of peers to upload the initial pieces to and that these peers are all in the same peer set, then they will have the same view of the rarest pieces, and they will download from the initial seed an entire copy of the content without any duplicate pieces. But, other peer selection policies may increase the ratio of duplicate pieces before a first copy of the content is sent. There is no such a problem with network coding. However, simple policies can be implemented to guarantee that the ratio of duplicate pieces remains low for the initial seed, e.g., the new choke algorithm in seed state or the super seeding mode [3]. In this case, the benefit of network coding compared to rarest first will not be significant at the scale of the content download.

Network coding appears as a solution more general than rarest first, as it works optimally in all cases. However, we argue in favor of the simplicity of rarest first. Network coding raises several implementation issues and is CPU intensive. Rarest first is simple, easy to implement, and already widely used. We have seen that in a context of peer-topeer content replication with a large peer set and a good network connectivity, rarest first is a simple and very efficient solution. That is in this context that we argue that a replacement of rarest first cannot be justified.

\subsection{Choke Algorithm}

The choke algorithm is a peer selection strategy. It should guarantee fairness and maximize the system capacity. In this section, we focus on the fairness issue, as the claimed deficiencies of the choke algorithm are related to its fairness properties. Whereas the evaluation and optimization of the system capacity is an important issue, the choke algorithm is indisputably an efficient peer selection strategy that is used by millions of persons. A detailed evaluation of the system capacity reached with the choke algorithm is an interesting area of future research.

\subsubsection{Fairness Issue}

Several recent studies $[5,10,13,15]$ challenge the fairness properties of the choke algorithm because it does not implement a bit level tit-for-tat, but a coarse approximation based on short term download estimations. Moreover, it is believed that a fair peer selection strategy must enforce a byte level reciprocation. For instance, a peer $A$ refuses to upload data to a peer $B$ if the amount of bytes uploaded by $A$ to $B$ minus the amount of bytes downloaded from $B$ to $A$ is higher than a given threshold $[5,10,15]$. The rationale behind this notion of fairness is that free riders should be penalized, and reciprocation should be enforced. We call this notion of fairness, tit-for-tat fairness.

We argue in the following that tit-for-tat fairness is not 
appropriate in the context of peer-to-peer file replication. A peer-to-peer session consists of seeds, leechers, and free riders, i.e., leechers that never upload data. We consider the free riders as a subset of the leechers. With tit-fortat fairness, when there is more capacity of service in the torrent than request for this capacity, the excess capacity will be lost even if slow leechers or free riders could benefit from it. Excess capacity is not rare as it is a fundamental property of peer-to-peer applications. Indeed, there are two important characteristics of peer-to-peer applications that tit-for-tat fairness does not take into account. First, leechers can have an asymmetrical network connectivity, the upload capacity being lower than the download capacity. In the case of tit-for-tat fairness, a leecher will never be able to use its full download capacity even if there is excess capacity in the peer-to-peer session. Second, a seed cannot evaluate the reciprocation of a leecher, because a seed does not need any piece. As a consequence, there is no way for a seed to enforce tit-for-tat fairness. But, seeds can represent an important part of a peer-to-peer session, see Table 1 For this reason, it is fundamental to have a notion of fairness that takes into account seeds.

In the following, we present two fairness criteria that take into account the characteristics of leechers and seeds and the notion of excess capacity:

- Any leecher $i$ with an upload speed $U_{i}$ should get a lower download speed than any other leecher $j$ with an upload speed $U_{j}>U_{i}$.

- A seed should give the same service time to each leecher.

With these two simple criteria, leechers are allowed to use the excess capacity, but not at the expense of leechers with a higher level of contribution. Reciprocation is fostered and free riders are penalized. Seeds do not make a distinction between contributing leechers and free riders. However, free riders cannot compromise the stability of the system because the more there are contributing leechers, the less the free riders receive from the seeds.

Tit-for-tat fairness can be extended to evenly distribute the capacity of seeds to peers in a torrent. With this extension, tit-for-tat fairness will verify our two fairness criteria. However, in the context of peers with asymmetric capacity, finding a threshold that maximizes the capacity of the system is a hard task that is not yet solved in the context of a distributed system. Moreover, using a default threshold may lead to a high inefficiency of the system. We will see in the following that the choke algorithm verifies our two fairness criteria with a simple distributed algorithm that does not require the complex computation of a threshold.

To summarize the above discussion, tit-for-tat fairness is not appropriate in the context of peer-to-peer file replication protocols like BitTorrent. For this reason, we proposed two new criteria of fairness, one for leechers and one for seeds. It is beyond the scope of this study to perform a detailed discussion of the fairness issues for peer-to-peer protocols. Our intent is to give a good intuition on how a peer-to-peer protocol should behave in order to achieve a reasonable level of fairness.

In the following, we show on real torrents that the choke algorithm in leecher state fosters reciprocation, and that the new choke algorithm in seed state gives the same service time to each leecher. We conclude that the choke algorithm is fair according to our two new fairness criteria.
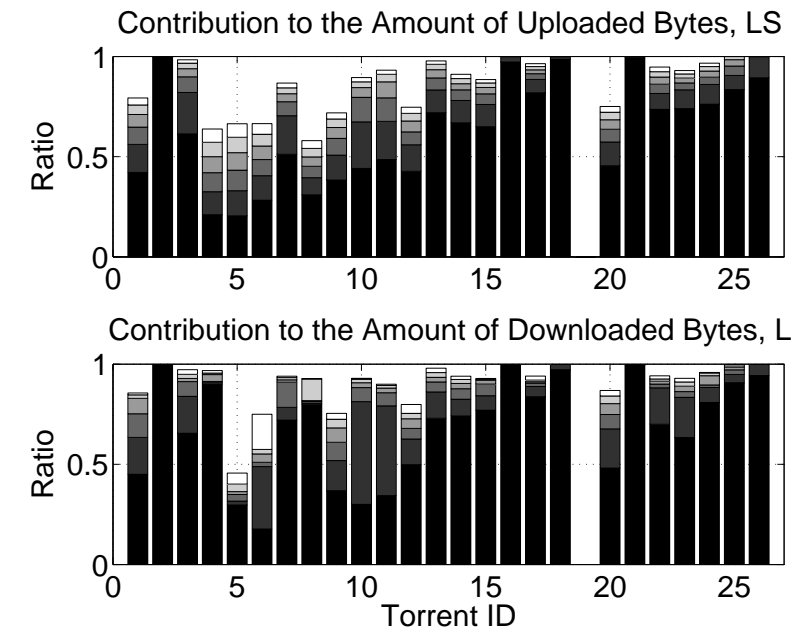

Figure 7: Fairness characterization of the choke algorithm in leecher state for each torrent. Top graph: Amount of bytes uploaded from the local peer to remote peers. We created 6 sets of 5 remote peers each, the first set (in black) contains the 5 remote peers that receive the most bytes from the local peer. Each next set contains the next 5 remote peers. The sets representation goes from black for the set containing the 5 best remote downloaders, to white for the set containing the 25 to 30 best downloaders. Bottom graph: Amount of bytes downloaded from remote peers to the local peer. The same set construction is kept. Thus, this graph shows how much each set of downloaders, as defined in the top graph, uploaded to the local peer.

\subsubsection{Leecher State}

The choke algorithm in leecher state fosters reciprocation. We see in Fig. 7 that peers that receive the most from the local peer (top graph) are also peers from which the local peer downloaded the most (bottom graph). Indeed, the same color in the top and bottom graphs represents the same set of peers. All seeds are removed from the data used for the bottom graph, as it is not possible to reciprocate data to seeds. This way, a ratio of 1 in the bottom graph represents the total amount of bytes downloaded from leechers.

Two torrents present a different characteristic. The local peer for torrent 19 does not upload any byte in leecher state because due to the small number of leechers in this torrent, the local peer in leecher state had no leecher in its peer set. Torrents 5, which is in transient state, has a low level of reciprocation. This is explained by a single leecher that gave to the local peer half of the pieces, but who received few pieces from the local peer. The reason is that this remote leecher was almost never interested in the local peer. This problem is due to the low entropy of the torrent in transient state.

Because the choke algorithm takes its decisions based on the current download rate of the remote peers, it does not achieve a perfect reciprocation of the amount of bytes downloaded and uploaded. However, Fig. 7 shows that the peers from which the local peer downloads the most are also the peers that receive the most uploaded bytes. Thus there is a strong correlation between the amount of bytes uploaded and the amount of bytes downloaded.

The above results show that with a simple distributed algorithm and without any stringent reciprocation require- 
Correlation Unchoke and Interested Duration, LS
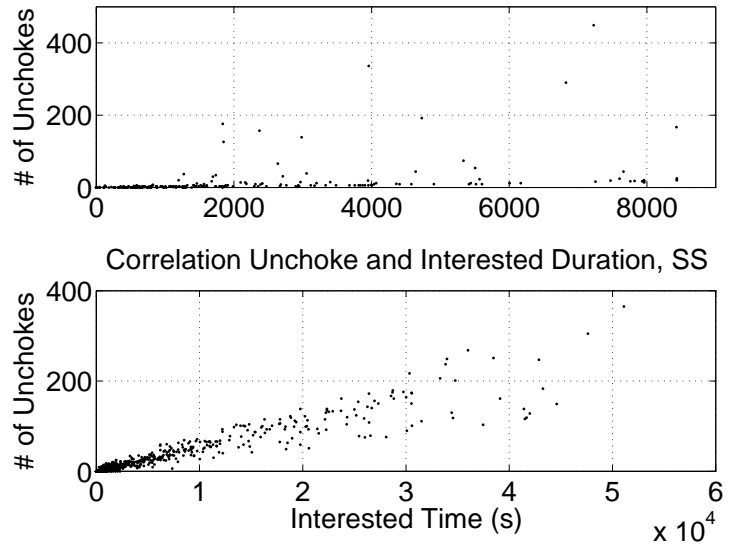

Figure 8: Correlation between the number of unchokes and the interested time for each remote peer for torrent 7 . For each remote peer, a dot represents the correlation between the number of times this remote peer is unchoked by the local peer and the time this remote peer is interested in the local peer. Top graph: Correlation when the local peer is in leecher state. Bottom graph: Correlation when the local peer is in seed state.

ments, unlike tit-for-tat fairness, one can achieve a good reciprocation. More importantly, the choke algorithm in leecher state allows leechers to benefit from the excess capacity. It is important to understand why the choke algorithm achieves this good reciprocation. One reason is the way the active peer set is built. In the following, we focus on how the local peer selects the remote peers to upload blocks to.

The choke algorithm in leecher state selects a small subset of peers to upload blocks to. We see in Fig. 7 top graph, that the 5 peers that receive the most data from the local peer (in black) represents a large part of the total amount of uploaded bytes. At first sight, this behavior is expected from the choke algorithm because a local peer selects the three fastest downloading peers to upload to, see section 2.3.2 However, there is no guarantee that these three peers will continue to send data to the local peer. In the case they stop sending data to the local peer, the local peer will also stop reciprocating to them.

We focus on torrent 7 in order to understand how this subset of peers is selected. Fig. 8 (top graph) shows that most of the peers are unchoked few times and few peers are unchoked frequently. The optimistic unchoke gives a chance to each peer to be unchoked few times, whereas the regular unchoke is used to unchoke frequently peers that send the fastest to the local peer. The optimistic unchoke acts as a peer discovery mechanism. The peers that are not unchoked at all are either initial seeds, or peers that do not stay in the peer set long enough to be optimistically unchoked.

We see in Fig. [ (top graph) that there is no correlation between the number of times a peer is unchoked and how long a peer is interested in the local peer. However, we see that the number of unchokes for the peers that are unchoked few times increases slightly with the interested time duration. This is because the optimistic unchoke takes at random a peer to be optimistically unchoked. Thus the longer a peer is interested in the local peer, the more likely it has to be optimistically unchoked.

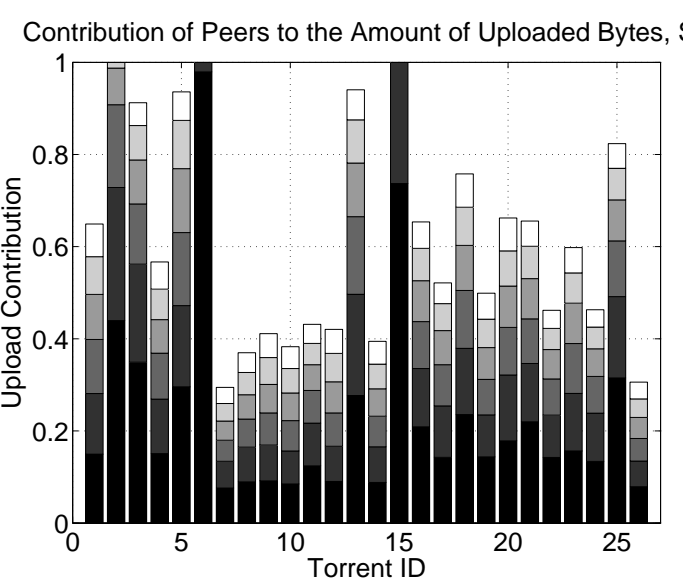

Figure 9: Fairness characterization of the choke algorithm in seed state for each torrent. Legend: We created 6 sets of 5 remote peers each, the first set (in black) contains the 5 remote peers that receive the most bytes from the local peer. Each next set contains the next 5 remote peers. The set representation goes from black from the set containing the 5 best remote downloaders, to white for the set containing the 25 to 30 best downloaders.

Fig. 17 shows that for four torrents in transient state, torrents 4, 5, 6 and 8, the amount of bytes uploaded by the 30 best remote peers is lower than for the other torrents. Torrents in transient state have low entropy. Therefore, the peers are no longer selected based only on their reciprocation level, but also on the pieces available. For this reason, a larger set of peers receives pieces from the local peer. Thus, a lower fraction of bytes uploaded to the best remote peers.

In summary, we have seen that the choke algorithm fosters reciprocation. One important reason is that each peer elects a small subset of peers to upload data to. This stability improves the level of reciprocation. We have seen that this stability is not due to a lack of interest. Our guess is that the choke algorithm leads to an equilibrium in the peer selection. The exploration of this equilibrium is fundamental to the understanding of the choke algorithm efficiency. It is beyond the scope of this study to do this analysis, but it is an important area of future research.

\subsubsection{Seed State}

The new choke algorithm in seed state gives the same service time to each remote peer. We see in Fig. 9] that each peer receives roughly the same amount of bytes from the local peer. The differences among the peers are due to the time remote peers are interested in the local peer. The more a remote peer is interested in the local peer, the more times this remote peer is unchoked. This is confirmed by Fig. 8 (bottom graph) that shows a strong correlation between the time a peer is interested in the local peer and the number of times the local peer unchokes it. For torrents 6 and 15 the five best downloaders receive most of the bytes, because for both torrents there were less than 10 remote peers that received bytes from the local peer.

This new version of the choke algorithm in seed state is the only one to give the same service time to each leecher. This has three fundamental benefits compared to the old version. First, as each leecher receives a small and equivalent service time from the seeds, the entropy of the pieces 
is improved. In contrast, with the old choke algorithm, a few fast leechers can receive most of the pieces, which decreases the diversity of the pieces. Second, free riders cannot receive more than contributing leechers. In contrast, with the old choke algorithm, a fast free rider can monopolize a seed. Third, the resilience in transient phase is improved. Indeed, the initial seed does not favor any leecher. Thus, if a leecher leaves the peer set, it will only remove a small subset of the pieces from the torrent. In contrast, with the old choke algorithm, the initial seed can send most of the pieces to a single leecher. If this leecher leaves the torrent, that will adversely impact the torrent and increase the time in transient state.

In summary, the new choke algorithm in seed state gives the same service to time to each leecher. This new algorithm is a significant improvement over the old one. In particular, whereas the old choke algorithm can be unfair and sensible to free riders, the new choke algorithm is fair and robust to free riders.

\section{RELATED WORK}

Whereas BitTorrent can be considered as one of the most successful peer-to-peer protocol, there are few studies on it.

Several analytical studies of BitTorrent-like protocols exist $[6,22,26]$. Whereas they provide a good insight into the behavior of such protocols, the assumption of global knowledge limits the scope of their conclusions. Biersack et al. [6] propose an analysis of three content distribution models: a linear chain, a tree, and a forest of trees. They discuss the impact of the number of chunks (what we call pieces) and of the number of simultaneous uploads (what we call the active peer set) for each model. They show that the number of chunks should be large and that the number of simultaneous uploads should be between 3 and 5. Yang et al. [26] study the service capacity of BitTorrent-like protocols. They show that the service capacity increases exponentially at the beginning of the torrent and then scale well with the number of peers. They also present traces obtained from a tracker. Such traces are very different from ours, as they do not allow to study the dynamics of a peer. Both studies presented in [6] and [26] are orthogonal to ours as they do not consider the dynamics induced by the choke and rarest first algorithms. Qiu and Srikant [22] extend the initial work presented in [26] by providing an analytical solution to a fluid model of BitTorrent. Their results show the high efficiency in terms of system capacity utilization of BitTorrent, both in a steady state and in a transient regime. Furthermore, the authors concentrate on a game-theoretical analysis of the choke and rarest first algorithms. However, a major limitation of this analytical model is the assumption of global knowledge of all peers to make the peer selection. Indeed, in a real system, each peer has only a limited view of the other peers, which is defined by its peer set. As a consequence, a peer cannot find the best suited peers to send data to in all the peers in the torrent (global optimization assumption), but in its own peer set (local and distributed optimization). Also, the authors do not evaluate the rarest first algorithm, but assume a uniform distribution of pieces. Our study is complementary, as it provides an experimental evaluation of algorithms with limited knowledge. In particular, we show that the efficiency on real torrents is close to the one predicted by the models.

Felber et al. [9] compare different peer and piece selection strategies in static scenarios using simulations. Bharambe et al. [5] present a simulation-based study of BitTorrent using a discrete-event simulator that supports up to 5000 peers. The authors concentrate on the evaluation of the BitTorrent performance by looking at the upload capacity of the nodes and at the fairness defined in terms of the volume of data served by each node. They varied various parameters of the simulation as the peer set and active peer set size. They provide important insights into the behavior of BitTorrent. However, they do not evaluate a peer set larger than 15 peers, whereas the real implementation of BitTorrent has a default value of 80 peers. This restriction may have an important impact on the behavior of the protocol as the piece selection strategy is impacted by the peer set size. The validation of a simulator is always hard to perform, and the simulator restrictions may biased the results. Our study provides real word results that can be used to validate simulated scenarios. Moreover, our study is different because we do not modify the default parameters of BitTorrent, but we observed its default behavior on a large variety of real torrents. Finally, we provide new insights into the rarest first piece selection and on the choke algorithm peer selection. In particular, we argue that the choke algorithm in its latest version is fair.

Pouwelse et al. [21] study the file popularity, file availability, download performance, content lifetime and pollution level on a popular BitTorrent tracker site. This work is orthogonal to ours as they do not study the core algorithms of BitTorrent, but rather focus on the contents distributed using BitTorrent and on the users behavior. The work that is the most closely related to our study was done by Izal et al. [14]. In this paper, the authors provide seminal insights into BitTorrent based on data collected from a tracker log for a single yet popular torrent, even if a sketch of a local vision from a local peer perspective is presented. Their results provide information on peers behavior, and show a correlation between uploaded and downloaded amount of data. Our work differs from [14] in that we provide a thorough measurement-based analysis of the rarest first and choke algorithms. We also study a large variety of torrents, which allows us not to be biased toward a particular type of torrent. Moreover, without pretending to answer all possible questions that arise from a simple yet powerful protocol as BitTorrent, we provide new insights into the rarest first and choke algorithms.

\section{DISCUSSION}

In this paper we go beyond the common wisdom that BitTorrent performs well. We have performed a detailed experimental evaluation of the rarest first and choke algorithms on real torrents with varying characteristics in terms of number of leechers, number of seeds, and content sizes. Whereas we do not pretend to have reached completeness, our evaluation gives a reasonable understanding of the behavior of both algorithms on a large variety of real cases.

Our main results are the following.

- The rarest first algorithm guarantees a close to ideal entropy on the presented torrents. In particular, it prevents the reappearance of rare pieces and of the last pieces problem.

- We have found that torrents in a startup phase can have low entropy. The duration of this phase depends only on the upload capacity of the source of the content. In particular, the rarest first algorithm is not responsible of the low entropy during this phase. 
- The fairness achieved with a bit level tit-for-tat strategy is not appropriate in the context of peer-to-peer file replication. We have proposed two new fairness criteria in this context.

- The choke algorithm is fair, fosters reciprocation, and is robust to free riders in its latest version.

Our main contribution is to show that on real torrents the rarest first and choke algorithms are enough to have an efficient and viable file replication protocol in the Internet. In particular, we discussed the benefits of the new choke algorithm in seed state. This new algorithm outperforms the old one and should replace it. We also identified two new areas of improvement: the downloading speed of the first blocks, and the duration of the transient phase.

The rarest first algorithm is simple. It does not require global knowledge or important computational resources. Yet, it guarantees a peer availability, for the peer selection, close to the ideal one. We do not see any striking argument in favor of a more complex solution in the evaluated context.

We do not claim that the choke algorithm is optimal. The understanding of its equilibrium is an area of future research. However, it achieves a reasonable level of efficiency, and most importantly it guarantees a viable system by fostering reciprocation, preventing free riders to attack the stability of the system, and using the excess capacity. Solutions based on a bit level tit-for-tat are not appropriate.

Our conclusions only hold in the context we explored, i.e., peer-to-peer file replication in the Internet. There are many different contexts where peer-to-peer file replication can be used: small files, small group of peers, dynamic groups in ad-hoc networks, peers with partial connectivity, etc. All these contexts are beyond the scope of this paper, but are interesting areas for future research.

We also identified two areas of improvement. The time to deliver the first blocks of data should be reduced. In the case of large contents, this delivery time will marginally increase the overall download time. But, in the case of small contents, the penalty is significant. Also, the duration of the transient phase should be minimized as the low entropy may results in a performance penalty. The way to solve these problems is beyond the scope of this study, but is an interesting area of future research.

We believe that this work sheds a new light on two new algorithms that enrich previous content distribution techniques in the Internet. BitTorrent is the only existing peerto-peer replication protocol that exploits these two promising algorithms in order to improve system capacity utilization. We deem that the understanding of these two algorithms is of fundamental importance for the design of future peer-to-peer content distribution applications.

\section{Acknowledgment}

We would like to thank the anonymous reviewers, and also Chadi Barakat, Ernst W. Biersack, Walid Dabbous, Katia Obraczka, Thierry Turletti for their valuable comments.

\section{REFERENCES}

[1] http://www.slyck.com

[2] http://www.bittorrent.com/

[3] Bittorrent protocol specification v1.0.

http://wiki.theory.org/BitTorrentSpecification June 2005.
[4] R. Bhagwan, S. Savagen, and G. Voelker. Understanding availability. In International Workshop on Peer-to-Peer Systems, Berkeley, CA, USA, February 2003.

[5] A. R. Bharambe, C. Herley, and V. N. Padmanabhan. Analysing and improving bittorrent performance. In Proc. IEEE Infocom'2006, Barcelona, Spain, April 2006.

[6] E. W. Biersack, P. Rodriguez, and P. Felber. Performance analysis of peer-to-peer networks for file distribution. In Proc. Fifth International Workshop on Quality of Future Internet Services (QofIS'04), Barcelona, Spain, September 2004.

[7] Y. Chawathe, S. Ratnasamy, L. Breslau, and S. Shenker. Making gnutella-like p2p systems scalable. In Proc. ACM SIGCOMM'03, Karlsruhe, Germany, August 25-29 2003.

[8] B. Cohen. Incentives build robustness in bittorrent. In Proc. First Workshop on Economics of Peer-to-Peer Systems, Berkeley, USA, June 2003.

[9] P. Felber and E. W. Biersack. Self-scaling networks for content distribution. In Proc. International Workshop on Self-* Properties in Complex Information Systems, Bertinoro, Italy, May-June 2004.

[10] P. Ganesan and M. Seshadri. On cooperative content distribution and the price of barter. In IEEE ICDCS'05, Columbus, Ohio, USA, June 2005.

[11] C. Gkantsidis and P. Rodriguez. Network coding for large scale content distribution. In Proc. IEEE Infocom'2005, Miami, USA, March 2005.

[12] K. Gummadi, R. Gummadi, S. Gribble, S. Ratnasamy, S. Shenker, and I. Stoica. The impact of dht routing geometry on resilience and proximity. In Proc. ACM SIGCOMM'03, Karlsruhe, Germany, August 25-29 2003.

[13] L. Guo, S. Chen, Z. Xiao, E. Tan, X. Ding, and X. Zhang. Measurements, analysis, and modeling of bittorrent-like systems. In Proc. ACM IMC'2005, Berkeley, CA, USA, October 2005.

[14] M. Izal, G. Urvoy-Keller, E. W. Biersack, P. Felber, A. A. Hamra, and L. Garcés-Erice. Dissecting bittorrent: Five months in a torrent's lifetime. In Proc. PAM'04, Antibes Juan-les-Pins, France, April 2004.

[15] S. Jun and M. Ahamad. Incentives in bittorrent induce free riding. In Proc. SIGCOMM'05 Workshops, Philadelphia, PA, USA, August 2005.

[16] T. Karagiannis, A. Broido, N. Brownlee, and K. C. Claffy. Is p2p dying or just hiding? In Proc. IEEE Globecom'04, Dalla, Texas, USA, Nov. 29-Dec. 32004.

[17] T. Karagiannis, A. Broido, M. Faloutsos, and K. C. Claffy. Transport layer identification of $\mathrm{p} 2 \mathrm{p}$ traffic. In Proc. ACM $I M C^{\prime} 04$, Taormina, Sicily, Italy, October 2004.

[18] D. Kostić, R. Braud, C. Killian, E. Vandekieft, J. W. Anderson, A. C. Snoeren, and A. Vahdat. Maintaining high bandwidth under dynamic network conditions. In Proc. USENIX'05, Anaheim, CA, USA, April 2005.

[19] A. Legout, G. Urvoy-Keller, and P. Michiardi. Rarest first and choke algorithms are enough. Technical Report (inria-00001111, version 3 - 6 September 2006), INRIA, Sophia Antipolis, September 2006.

[20] A. Parker. The true picture of peer-to-peer filesharing. http://www.cachelogic.com/ July 2004.

[21] J. A. Pouwelse, P. Garbacki, D. H. J. Epema, and H. J. Sips. The bittorrent $\mathrm{p} 2 \mathrm{p}$ file-sharing system: Measurements and analysis. In Proc. 4th International Workshop on Peer-to-Peer Systems (IPTPS'05), Ithaca, New York, USA, February 2005.

[22] D. Qiu and R. Srikant. Modeling and performance analysis of bittorrent-like peer-to-peer networks. In Proc. ACM SIGCOMM'04, Portland, Oregon, USA, Aug. 30-Sept. 32004.

[23] S. Ratnasamy, P. Francis, M. Handley, R. Karp, and S. Shenker. A scalable content-addressable network. In Proc. ACM SIGCOMM'01, San Diego, California, USA, August 27-31 2001.

[24] P. Rodriguez and E. W. Biersack. Dynamic parallel-access to replicated content in the internet. IEEE/ACM Transactions on Networking, 10(4), August 2002.

[25] I. Stoica, R. Morris, D. Karger, M. F. Kaashoek, and H. Balakrishnan. Chord: A scalable peer-to-peer lookup service for internet applications. In Proc. ACM SIGCOMM'01, San Diego, California, USA, August 27-31 2001.

[26] X. Yang and G. de Veciana. Service capacity in peer-to-peer networks. In Proc. IEEE Infocom'04, pages 1-11, Hong Kong, China, March 2004. 